\title{
The impact of severe haemophilia and the presence of target joints on health-related quality-of-life
}

\author{
Jamie O'Hara ${ }^{1,2}$, Shaun Walsh², Charlotte Camp ${ }^{2,6^{*}}$, Giuseppe Mazza ${ }^{3}$, Liz Carroll ${ }^{4}$, Christina Hoxer ${ }^{5}$ \\ and Lars Wilkinson ${ }^{5}$
}

\begin{abstract}
Background: Joint damage remains a major complication associated with haemophilia and is widely accepted as one of the most debilitating symptoms for persons with severe haemophilia. The aim of this study is to describe how complications of haemophilia such as target joints influence health-related quality of life (HRQOL).

Methods: Data on hemophilia patients without inhibitors were drawn from the 'Cost of Haemophilia across Europe - a Socioeconomic Survey' (CHESS) study, a cost-of-illness assessment in severe haemophilia A and B across five European countries (France, Germany, Italy, Spain, and the UK). Physicians provided clinical and sociodemographic information for 1285 adult patients, 551 of whom completed corresponding questionnaires, including EQ-5D.

A generalised linear model was developed to investigate the relationship between EQ-5D index score and target joint status (defined in the CHESS study as areas of chronic synovitis), adjusted for patient covariates including socio-demographic characteristics and comorbidities.
\end{abstract}

Results: Five hundred and fifteen patients (42\% of the sample) provided an EQ-5D response; a total of 692 target joints were recorded across the sample. Mean EQ-5D index score for patients with no target joints was 0.875 (standard deviation [SD] 0.179); for patients with one or more target joints, mean index score was 0.731 (SD 0. 285). Compared to having no target joints, having one or more target joints was associated with lower index scores (average marginal effect (AME) -0.120; SD 0.0262; $p<0.000$ ).

Conclusions: This study found that the presence of chronic synovitis has a significant negative impact on HRQOL for adults with severe haemophilia. Prevention, early diagnosis and treatment of target joints should be an important consideration for clinicians and patients when managing haemophilia.

Keywords: Haemophilia, Disease burden, Joint disease, Health-related quality of life, Patient-reported outcome measures

\section{Background}

Haemophilia is a rare, lifelong bleeding disorder characterised by a deficiency of coagulation factor, with an estimated incidence of the two most common forms (Haemophilia A, a Factor VIII deficiency; and Haemophilia B, a Factor IX deficiency) of 1 per 4000-5000 and 1 per 20,000, respectively [1,2]. Approximately $70 \%$ of cases arise via $\mathrm{X}$-linked recessive inheritance; the

\footnotetext{
* Correspondence: charlotte.camp@hcdeconomics.com

${ }^{2} \mathrm{HCD}$ Economics, Daresbury, UK

${ }^{6}$ HCD Economics, The Innovation Centre, Daresbury WA4 4FS, UK

Full list of author information is available at the end of the article
}

remaining 30\% arise with no known familial history ('sporadic' haemophilia) [3]. Its primary manifestation is prolonged bleeding of the musculoskeletal system and, less frequently, mucosal and cerebral haemorrhages; persons with severe (coagulation factor $<1 \%$ of normal) haemophilia are particularly prone to spontaneous bleed events (occurring in the absence of notable trauma).

Approximately $80 \%$ of bleed events are intra-articular in nature, two-thirds of which are reported in the knees, elbows, and ankles [4]. Bleed events lead to swelling and acute pain of the affected area, alleviated through infusions of plasma-derived or recombinant coagulation

(c) The Author(s). 2018 Open Access This article is distributed under the terms of the Creative Commons Attribution 4.0 International License (http://creativecommons.org/licenses/by/4.0/), which permits unrestricted use, distribution, and reproduction in any medium, provided you give appropriate credit to the original author(s) and the source, provide a link to the Creative Commons license, and indicate if changes were made. The Creative Commons Public Domain Dedication waiver (http://creativecommons.org/publicdomain/zero/1.0/) applies to the data made available in this article, unless otherwise stated. 
factor and respite care [5]. Frequent bleed events (2-3 in a six-month period) to the same joint site is associated with chronic inflammation of the synovium and reduced joint flexion and mobility, arising as a result of increased volume of fluid within the joint [6]. In the absence of appropriate management, chronic synovitis is a significant risk factor for long-term deterioration of the joint via haemophilic arthropathy, leading to chronic pain, joint disfigurement, and disability [7-9].

With the advent of prophylactic factor concentrate regimens, introduced in Europe in the 1980s, the majority of persons with haemophilia (PWH) aged 30 and under exhibit minimal joint damage relative to that of previous generations [10]. However, risk of inhibitor development, whereby an autoimmune response to factor concentrate renders the product ineffective, is a significant risk for PWH, particularly for newly diagnosed infants in the early days of therapy exposure and those undergoing surgery [11]. In the absence of effective treatment, either with bypass therapies or through 'training' the body to accept factor concentrate ('immune tolerance induction' or ITI), the presence of an inhibitor can significantly increase bleed frequency and accelerate joint damage [12].

The psychological burden of degenerative, heritable diseases such as haemophilia is an important yet historically overlooked aspect of economic evaluations and health policy considerations, which more often focus on disease-related morbidity and mortality [13]. However, as major therapeutic advances in haemophilia have extended life expectancy and improved clinical outcomes for sufferers, more recent studies have shifted focus to health-related quality of life (HRQOL) as an outcome measure, either with generic or disease-specific instruments $[14,15]$. However, the specific impact of musculoskeletal complications of haemophilia has not so far been considered in isolation.

The purpose of this paper is to utilise a recent (2015) observational study in severe haemophilia across five European countries to explore drivers of HRQOL. Specifically, the analysis will explore the relationship between target joints and HRQOL for severe PWH, and the extent to which patient-reported health and wellbeing is driven by long-term clinical outcomes. While this topic has been explored to some detail within single-country studies, the use of data from the 'Cost of Haemophilia in Europe - a Socioeconomic Survey' (CHESS) dataset affords access to one of the largest samples of patient-reported HRQOL of recent years.

\section{Methods}

\section{Data source}

CHESS is a retrospective, non-interventional study of severe, inherited haemophilia A and B across five European countries (France, Germany, Italy, Spain, and the UK).
The purpose of the study was to generate an annualised economic burden of the condition through reporting of 12-month, haemophilia-related direct and indirect resource use [16]. One hundred and thirty-nine haematologists provided demographic and clinical information for 1285 adult ( $\geq 18$ years) patients, 541 (42\%) of whom completed a corresponding questionnaire covering out-ofpocket medical expenditure, HRQOL (measured using the EQ-5D-3 L) and work loss.

Study exclusion criteria was limited to patients diagnosed with an inhibitor at the time of study capture $(n=52)$, due to a differing risk profile for bleeds and

Table 1 Patient characteristics $(n=515)$

\begin{tabular}{|c|c|}
\hline Age (mean $\pm S D)$ & $37.7 \pm 15.0$ \\
\hline \multicolumn{2}{|l|}{ Age categories (\%) } \\
\hline $18-30$ & $205(39.8 \%)$ \\
\hline $31-40$ & $119(62.9 \%)$ \\
\hline $41-50$ & 89 (17.3\%) \\
\hline $51-60$ & $50(9.7 \%)$ \\
\hline $61+$ & $52(10.1 \%)$ \\
\hline \multicolumn{2}{|l|}{ Subtype (\%) } \\
\hline Haemophilia A & $400(77.7 \%)$ \\
\hline Haemophilia B & $115(22.3 \%)$ \\
\hline \multicolumn{2}{|l|}{ Country (\%) } \\
\hline France & $180(35.0 \%)$ \\
\hline Germany & $96(18.6 \%)$ \\
\hline Italy & $118(22.9 \%)$ \\
\hline Spain & $84(16.3 \%)$ \\
\hline UK & $37(7.2 \%)$ \\
\hline \multicolumn{2}{|l|}{ Treatment Strategy (\%) } \\
\hline On-demand & $201(39.0 \%)$ \\
\hline Primary prophylaxis & $314(61.0 \%)$ \\
\hline \multicolumn{2}{|l|}{ Physician-reported comorbidities } \\
\hline Depression & 77 (15.0\%) \\
\hline Anxiety & $84(16.5 \%)$ \\
\hline Target joints (total) & 714 \\
\hline Target joints (mean \pm SD (range)) & $1.39 \pm 1.44(0-9)$ \\
\hline \multicolumn{2}{|c|}{ Number of target joints (patient n, \%) } \\
\hline Zero & $157(30.5 \%)$ \\
\hline One & $153(29.7 \%)$ \\
\hline Two & $130(25.2 \%)$ \\
\hline Three or more & $75(14.6 \%)$ \\
\hline \multicolumn{2}{|c|}{ Location of target joints (patient n, \%) } \\
\hline Exclusively upper body & $92(25.7 \%)$ \\
\hline Exclusively lower body & $188(52.5 \%)$ \\
\hline Upper and lower body & $78(21.8 \%)$ \\
\hline
\end{tabular}

Note. Values are means \pm SD or numbers (\%) 
subsequent target joint development among these patients $[12,17]$.

\section{EQ-5D}

The EQ-5D is one of the most frequently used generic tools for assessing HRQOL [18]. The questionnaire consists of two sections, the first of which (health state) consists of five dimensions (mobility, self-care, usual activities, pain/discomfort, and anxiety/depression), with responses rated on an ordinal (1-3) scale. For each of the five dimensions, the respondent indicates the statement best describing their 'health state' at that time, with 1 equivalent to 'no issues' and 3 to 'severe issues'. A health state index 'utility' score based on country-specific 'value sets' is derived through an amalgam of the five responses, with scores generally ranging from 0 (equivalent to 'dead') to 1 ('perfect health'), though scores of less than zero (states 'worse than dead') can also be derived [19].

The second part of the EQ-5D is a visual analogue scale (VAS). The respondent is asked to indicate how good or bad their health is on that day, based on a scale from 0 to 100 , where zero represents 'the worst health' the respondent can imagine, and 100 'the best health state' the person can imagine. Corresponding country population norms for both the EQ-5D index score and the VAS were used in this analysis, with the exception of Italy, for which the Spanish value set was used.

\section{Target joint definition}

A 'target joint' as defined in the CHESS study encompasses any joint with known chronic synovitis; in contrast to previous clinical studies [20], study investigators were given discretion as to how this may be further defined with respect to bleed frequency and period of observation. In order to explore the differential impact of costs associated with lower and upper body joint deterioration, target joints were categorised into two groups based on their location. 'Upper body' target joints were those in the shoulders, elbows, wrists, neck, and spine; 'lower body' target joints consisted of hips, knees, and ankles. The target joint variable was assessed in three ways: as a binary $0 / 1$ variable; as a binary $0 / 1$ variable split into upper and lower body joints; and as a discrete variable.

\section{Statistical analysis}

Demographic data and EQ-5D index score and VAS responses were compared between the sample of patients with no reported target joints and those with one or more reported target joints. Means were used to describe continuous variables; categorical variables are described as frequencies and proportions. Standard t-tests were conducted in order to test for between-group differences.

The marginal effect of the presence of one or more target joints on EQ-5D-3 L index score was assessed using a generalized linear model (GLM). Health utility data on a 'poor'-'good' scale is often negatively skewed with a large volume of unity values (i.e. perfect HRQOL) and a long 'tail' from a select group of less healthy 'outlier' patients. The GLM is an extension of the linear regression framework (Eq. 1) suitable for nonparametric outcome variables [21, 22]. The GLM requires a link function relating the conditional mean to the covariates, and a distribution 'family' to specify the relationship between the variance and the mean [23]. The log-link function (Eq. 2) in combination with a Poisson distribution (Eq. 3) is frequently used to model EQ-5D index scores and was employed for this analysis [24]. Index scores were transformed to a 'disutility' index score $(\mathrm{nY}=1-\mathrm{Y})$ in order to fit the model specification $[25,26]$. A confirmatory analysis of the family and link functions was conducted using the modified Park test $[21,22]$.

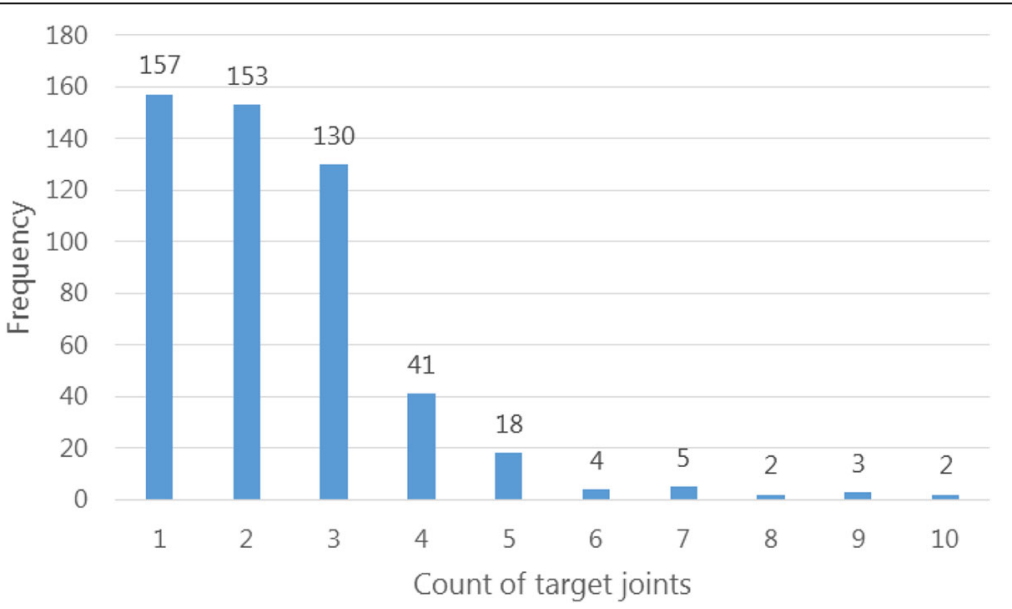

Fig. 1 Distribution of study cohort by count of target joints $(N=515)$ 
Table 2 EQ-5D-3 L index score by target joint status

\begin{tabular}{|c|c|c|c|}
\hline & $\begin{array}{l}\text { Total } \\
(n=515)\end{array}$ & $\begin{array}{l}\text { No target joints } \\
(n=157)\end{array}$ & $\begin{array}{l}\geq 1 \text { target joints } \\
(n=358)\end{array}$ \\
\hline Total & $0.77 \pm 0.27$ & $0.87 \pm 0.01$ & $0.73 \pm 0.29$ \\
\hline \multicolumn{4}{|l|}{ Age categories } \\
\hline $18-30$ & $0.86 \pm 0.01$ & $0.94 \pm 0.01$ & $0.82 \pm 0.21$ \\
\hline $31-40$ & $0.78 \pm 0.23$ & $0.84 \pm 0.16$ & $0.74 \pm 0.25$ \\
\hline $41-50$ & $0.72 \pm 0.28$ & $0.86 \pm 0.12$ & $0.69 \pm 0.30$ \\
\hline $51-60$ & $0.67 \pm 0.34$ & $0.83 \pm 0.19$ & $0.63 \pm 0.36$ \\
\hline $61+$ & $0.61 \pm 0.36$ & $0.73 \pm 0.31$ & $0.54 \pm 0.36$ \\
\hline \multicolumn{4}{|l|}{ Subtype } \\
\hline Haemophilia A & $0.78 \pm 0.26$ & $0.87 \pm 0.18$ & $0.74 \pm 0.28$ \\
\hline Haemophilia B & $0.76 \pm 0.29$ & $0.86 \pm 0.18$ & $0.70 \pm 0.32$ \\
\hline \multicolumn{4}{|l|}{ Country } \\
\hline France & $0.75 \pm 0.28$ & $0.87 \pm 0.18$ & $0.69 \pm 0.30$ \\
\hline Germany & $0.90 \pm 0.12$ & $0.93 \pm 0.09$ & $0.88 \pm 0.13$ \\
\hline Italy & $0.85 \pm 0.12$ & $0.86 \pm 0.14$ & $0.84 \pm 0.11$ \\
\hline Spain & $0.66 \pm 0.34$ & $0.71 \pm 0.36$ & $0.65 \pm 0.33$ \\
\hline UK & $0.59 \pm 0.36$ & $0.78 \pm 0.17$ & $0.56 \pm 0.38$ \\
\hline \multicolumn{4}{|l|}{ Treatment strategy } \\
\hline On-demand & $0.80 \pm 0.26$ & $0.87 \pm 0.21$ & $0.77 \pm 0.27$ \\
\hline Prophylaxis & $0.75 \pm 0.01$ & $0.87 \pm 0.01$ & $0.71 \pm 0.29$ \\
\hline \multicolumn{4}{|c|}{ Physician reported comorbidities } \\
\hline Depression & $0.60 \pm 0.36$ & $0.79 \pm 0.33$ & $0.55 \pm 0.35$ \\
\hline Anxiety & $0.80 \pm 0.26$ & $0.87 \pm 0.21$ & $0.77 \pm 0.27$ \\
\hline \multicolumn{4}{|c|}{ Number of target joints (patient n, \%) } \\
\hline One & - & - & $0.76 \pm 0.28$ \\
\hline Two & - & - & $0.76 \pm 0.26$ \\
\hline Three or more & - & - & $0.62 \pm 0.31$ \\
\hline \multicolumn{4}{|c|}{ Location of target joints (patient n, \%) } \\
\hline Exclusively upper body & - & - & $0.77 \pm 0.27$ \\
\hline Exclusively lower body & - & - & $0.73 \pm 0.28$ \\
\hline Upper and lower body & - & - & $0.68 \pm 0.31$ \\
\hline
\end{tabular}

$$
N D D C s_{12 m t h}=\alpha+\beta_{1}(\text { target joints })+\beta_{2} x_{i}+\ldots+\beta_{n} x_{n}
$$

Where $i=1, \ldots, n$

$$
\begin{aligned}
& E[y \mid x]=f\left(x^{\prime} \beta\right)=\exp \left(x^{\prime} \beta\right) \operatorname{In}(E[y \mid x])=x^{\prime} \beta \\
& y \sim \operatorname{Var}(y \mid x) \approx(E[y \mid x])^{\lambda}
\end{aligned}
$$

Additional model covariates were country of residence, patient age, and physician-reported presence of mental illness (anxiety and/or depression) via a stepwise inclusion method. Results are presented as average mean effects (AME). All statistical analysis was conducted using Stata 13 [27].

\section{Results}

Patient characteristics

The average age of the patient cohort was 37.7 years old, with the majority of patients (62.9\%) aged between 31 and 40 years (Table 1$)$. Almost two-thirds (61.0\%) of patients were receiving therapy via a prophylaxis regimen at the time of the study.

A total of 714 target joints were recorded across the study population (mean 1.39; SD 1.44; range 0-9) (Fig. 1). Three hundred and fifty-eight patients (69.5\%) were reported diagnosed with one or more target joints, with the majority (79\%) diagnosed with one or two target joints. The majority $(52.5 \%)$ of patients had target joints exclusively in the lower body.

\section{Factors influencing $\mathrm{HRQOL}$}

The mean EQ-5D index score in the sample was 0.77 (SD 0.27) (Table 2). Age was found to have a negative impact on HRQOL: index scores were found to decrease as patients progressed into each 10-year age cohort. Patients from Germany had the highest index score (mean 0.90; SD 0.12) and the United Kingdom the lowest

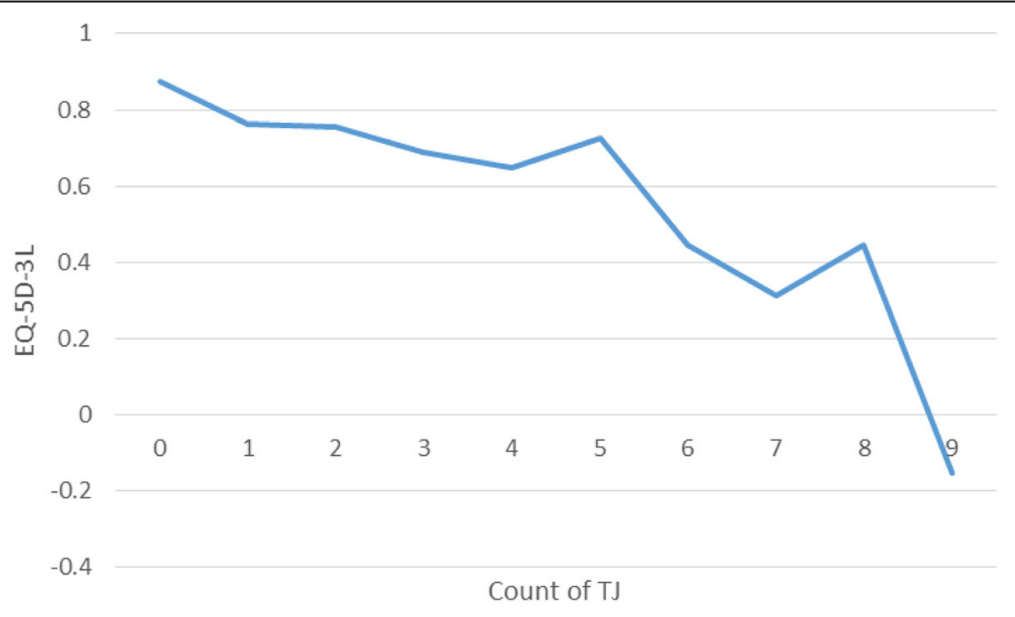

Fig. 2 EQ-5D-3 $L$ index score by number of target joints $(N=515)$ 


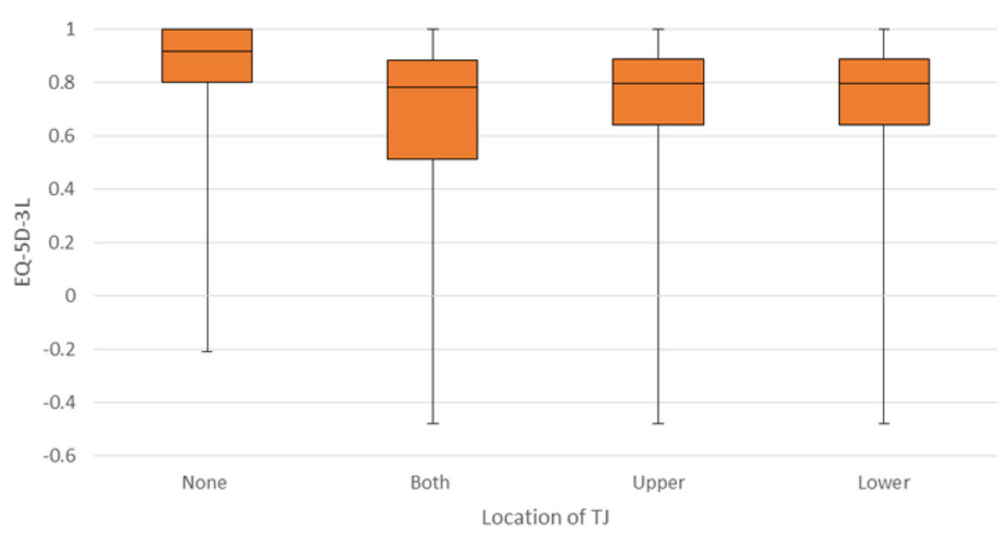

Fig. 3 EQ-5D-3 L index score by location of target joint

scores (mean 0.59, SD 0.37). Patients receiving prophylaxis had lower mean index scores compared to ondemand (mean 0.80 versus 0.75 ).

Patients with no recorded target joints had significantly higher utilities than those with one or more target joints (mean 0.87 versus 0.73 ), with scores deteriorating as the number of target joints increased (Fig. 2). Index scores among patients with an upper body target joint was broadly similar to those with a lower body target joint (mean 0.77 versus 0.73 ). Patients with both an upper and lower body target joint had lower index scores versus those with target joints in one location (upper or lower body) (mean index score 0.68, SD 0.31) (Fig. 3).

\section{Relationship between target joint status and EQ-5D VAS} Patients with no target joints reported the highest VAS scores (mean 74.3, SD 0.9) (Table 3), while patients with both an upper and lower body target joint reported the lowest VAS scores (mean 64.4, SD 18.3). VAS scores among patients with an upper body target joint were identical to those with a lower body target joint (mean 67.9). Mean reported VAS scores followed a downward trend as the number of target joints increased (Fig. 4).

\section{Individual dimensions of the EQ-5D-3 L}

Across all five dimensions of the EQ-5D-3 L, the majority of patients reported no problems, with fewer than one in ten patients reporting 'extreme' problems in any dimension (Table 4). Across the cohort as a whole, as well as within the cohort of patients with target joints, pain/discomfort and anxiety/depression were the most common dimensions in which 'extreme' problems were reported. The cohort of patients with one or more target joints consistently reported a higher frequency of extreme problems compared to those with no target joints: whether that be in mobility $(0.0 \%$ versus $1.4 \%)$; self-care
(0.6\% versus $2.0 \%)$; usual activities ( $0.6 \%$ versus $2.8 \%)$; pain/discomfort (1.3\% versus $4.7 \%)$; and anxiety/depression $(3.2 \%$ versus $5.6 \%)$.

\section{Multivariate analysis}

The presence of one or more target joints was associated with lower EQ-5D index scores (AME -0.120; SD 0.026; $p<0.001$ ) (Table 5). Patients in Germany or Italy with one or more target joints had the highest index scores (AME 0.110 and 0.121 respectively; $p<0.001$ ); patients in the UK were found to have the lowest mean index scores (AME -0.154, SD 0.060). The AME of patient age on index scores was minimal yet significant (mean $0.005 ; \mathrm{SD} 0.001 ; \mathrm{p}<0.001$ ). Anxiety/depression was found to be associated with lower index scores (AME -0.106; SD 0.001; $\mathrm{p}<0.001)$.

\section{Discussion and conclusion}

The findings of this analysis suggest an association between musculoskeletal complications of haemophilia and HRQOL: patients with no recorded target joints had the highest mean EQ-5D index and VAS scores; further, the number of target joints was inversely

Table 3 EQ-5D VAS score by target joint status

Total $69.3 \pm 17.0$

Number of target joints (patient $n, \%$ )

Zero

$74.3 \pm 0.9$

One

$69.1 \pm 15.7$

Two

$67.1 \pm 17.5$

Three or more

$63.1 \pm 16.5$

Location of target joints (patient $n, \%$ )

Exclusively upper body

$67.9 \pm 15.0$

Exclusively lower body

$67.9 \pm 16.7$

Upper and lower body

$64.4 \pm 18.3$

Note: Values are means \pm SD 


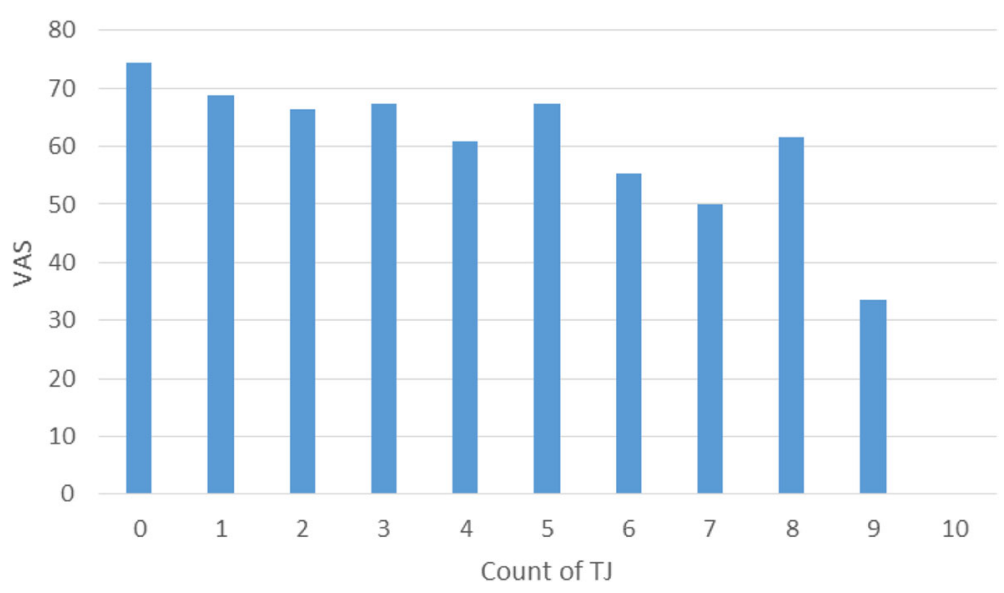

Fig. 4 EQ-5D VAS score by count of target joints

correlated with both measures. There was limited difference in index scores for individuals with either an upper or lower body target joint; patients with target joints in both locations had the lowest HRQOL within both the health state index score and VAS score. The results of the multivariate analysis show that, despite the confounding influences of age and nationality, the presence of one or

Table 4 Frequencies of item responses in each EQ-5D health state dimension by target joint status (\%)

\begin{tabular}{|c|c|c|c|}
\hline \multirow[b]{2}{*}{ Dimension } & \multicolumn{3}{|c|}{ Target joint status } \\
\hline & $\begin{array}{l}\text { All } \\
(n=515)\end{array}$ & $\begin{array}{l}\text { No target joints } \\
(n=157)\end{array}$ & $\begin{array}{l}\geq 1 \text { target joints } \\
(n=358)\end{array}$ \\
\hline \multicolumn{4}{|l|}{ Mobility } \\
\hline No problems & $312(60.6 \%)$ & 114 (72.6\%) & $198(55.3 \%)$ \\
\hline Some & $198(38.4 \%)$ & $43(27.4 \%)$ & $155(43.3 \%)$ \\
\hline Extreme & $5(1.0 \%)$ & $0(0.0 \%)$ & $5(1.4 \%)$ \\
\hline \multicolumn{4}{|l|}{ Self-care } \\
\hline No problems & $402(78.1 \%)$ & 135 (86\%) & $267(74.6 \%)$ \\
\hline Some & $105(20.4 \%)$ & $21(13.4 \%)$ & 84 (23.5\%) \\
\hline Extreme & $8(1.6 \%)$ & $1(0.6 \%)$ & $7(2.0 \%)$ \\
\hline \multicolumn{4}{|l|}{ Usual activities } \\
\hline No problems & $343(66.6 \%)$ & 119 (75.8\%) & $224(62.6 \%)$ \\
\hline Some & $161(31.3 \%)$ & 37 (23.6\%) & $124(34.6 \%)$ \\
\hline Extreme & $11(2.1 \%)$ & $1(0.6 \%)$ & $10(2.8 \%)$ \\
\hline \multicolumn{4}{|l|}{ Pain/Discomfort } \\
\hline No problems & $205(39.8 \%)$ & $95(60.5 \%)$ & $110(30.7 \%)$ \\
\hline Some & $291(56.5 \%)$ & 60 (38.2\%) & $231(64.5 \%)$ \\
\hline Extreme & $19(3.7 \%)$ & $2(1.3 \%)$ & $17(4.7 \%)$ \\
\hline \multicolumn{4}{|c|}{ Anxiety/Depression } \\
\hline No problems & $308(59.8 \%)$ & $113(72.0 \%)$ & $195(54.5 \%)$ \\
\hline Some & $182(35.3 \%)$ & $39(24.8 \%)$ & $143(39.9 \%)$ \\
\hline Extreme & 25 (4.9\%) & $5(3.2 \%)$ & $20(5.6 \%)$ \\
\hline
\end{tabular}

more target joints remained a significant driver of reduced HRQOL among our study cohort.

The long-term complications of haemophilia can have pronounced effects on HRQOL early in life - due to anxiety over future problems - and later in life, when the complications manifest themselves [28-30]. The analysis supports previous studies which suggest haemophilia patients experience depression and anxiety more often than the general population of a similar age [31]. Fatigue, loss of enjoyment, and reduced leisure pursuits also seem to correlate positively with the presence of target joints [28-30]. The pain and discomfort dimension of the EQ-5D-3 L was found to be particularly sensitive to the presence of target joints, with more than eight times as many patients reporting extreme pain or discomfort when a target joint was recorded.

Table 5 Multivariate Poisson regression analyses of disutility derived from EQ-5D index scores

\begin{tabular}{lll}
\hline & Model 1 & Model 2 \\
\hline Country & & \\
France & Omitted \\
Germany & & $-0.110^{\mathrm{a}}(0.025)$ \\
Italy & $-0.121^{\mathrm{a}}(0.022)$ \\
Spain & & $0.029^{\mathrm{a}}(0.034)$ \\
UK & & $0.154(0.060)$ \\
Age & & $0.005^{\mathrm{b}}(0.001)$ \\
Mental illness & & $0.106^{\mathrm{b}}(0.001)$ \\
Has target joint & & $0.120^{\mathrm{a}}(0.026)$ \\
$\quad$ AIC & $0.173^{\mathrm{a}}(0.031)$ & 0.956 \\
$\quad$ BIC & 1.002 & -3068.135 \\
\hline
\end{tabular}

Note: Results shown are average mean effect (AME) of each covariate on EQ-5D 'disutility' (1 - index score). Standard errors are shown in brackets

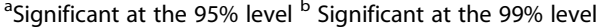

'Physician-reported anxiety or depression 
Our study has several limitations. The definition of a target joint in this analysis differs from current European guidelines that focus on bleed frequency as a measure of target joint status [32]. While the definitions exhibit significant overlap, reporting and observance of a bleed event is more straightforward than obtaining a clinical diagnosis of chronic synovitis, which often requires ultrasound and/or MRI assessment [33]. As discussed elsewhere [34], the definition of a target joint used in the CHESS study encompasses both these standardized definitions based on bleed rates and a consideration for longer-term degenerative changes to the joint tissue and structure arising from repeat bleed events.

Further, while our analysis attempts to create a comprehensive model of the main factors influencing EQ-5D index scores in severe haemophilia, there is the potential that some drivers were not accounted for, such as BMI, $\mathrm{HIV} / \mathrm{HCV}$ seropositivity, and sociodemographic factors such as employment and marital status [28, 30, 31, 35]. Our choice of HRQOL measure, though used widely, also presents limitations with respect to sensitivity and discriminatory power, particularly in comparison to the more recent five-level $(5 \mathrm{~L})$ version [36].

Nevertheless, the results presented in this study suggest that chronic synovitis in severe haemophilia is associated with reduced HRQOL as measured by the EQ-5D. Approaches to minimising the long-term risk of joint damage and deterioration among these patients - beginning at a young age with proactive therapy protocols to minimise bleed frequency and severity - will serve to reduce future psychosocial burdens on patients and are a justification for continued access to preventative therapy protocols. Further studies should seek to combine the cost and HRQOL consequences of bleed events and joint disease, and hence to quantify the cost effectiveness of current therapy protocols for severe haemophilia.

Our analysis demonstrates that haemophilia-related medical complications, such as the presence of one or more target joints, can have a major impact on HRQOL for persons with severe haemophilia.

\section{Acknowledgements}

Kind thanks to lan Jacob (HCD Economics) for reviewing the manuscript drafts.

\section{Funding}

This study was funded by Novo Nordisk. Publication was not contingent upon study results.

\section{Availability of data and materials}

The datasets generated and/or analysed during the current study are held under license by the University of Chester and are not publicly available, but are available from the corresponding author on reasonable request.

\section{Authors' contributions}

$\mathrm{JOH}$ designed the study protocol. CC and SW analysed the data. JOH, SW, and CC wrote the manuscript. LC and GM provided non-clinical perspective for the analysis and manuscript. All authors reviewed the manuscript. All authors read and approved the final manuscript.

\section{Ethics approval and consent to participate}

The CHESS study was reviewed by the Research Ethics Sub Committee of the Faculty of Health and Social Care within the University of Chester. Patient consent for use of clinical data was not required (as per European Pharmaceutical Market Research Association (EPhMRA) guidelines). Patient consent was obtained via tick box selection for the PSC element of the study.

\section{Competing interests}

LW and $\mathrm{CH}$ are employees of Novo Nordisk.

\section{Publisher's Note}

Springer Nature remains neutral with regard to jurisdictional claims in published maps and institutional affiliations.

\section{Author details \\ ${ }^{1}$ Faculty of Health and Social Care, University of Chester, Chester, UK. ${ }^{2} \mathrm{HCD}$ Economics, Daresbury, UK. ${ }^{3}$ UCL Institute for Liver and Digestive Health, Royal Free Hospital, University College London, London, UK. ${ }^{4}$ The Haemophilia Society, London, UK. ${ }^{5}$ Novo Nordisk A/S, Vandtårnsvej 114 DK-2860 Søborg, Denmark. ${ }^{6} \mathrm{HCD}$ Economics, The Innovation Centre, Daresbury WA4 4FS, UK}

Received: 16 August 2017 Accepted: 20 April 2018

Published online: 02 May 2018

\section{References}

1. Peyvandi F, Jayandharan G, Chandy M, Srivastava A, Nakaya SM, Johnson MJ, et al. Genetic diagnosis of haemophilia and other inherited bleeding disorders. Haemophilia. 2006;12:82-9.

2. Philip J, Sarkar RS, Kumar S, Prathip BR, Pathak A. Factor IX deficiency (Christmas disease). Med J Armed Forces India. 2012;68:379-80.

3. Kasper CK, Lin JC. Prevalence of sporadic and familial haemophilia. Haemophilia. 2007;13:90-2

4. Lobet S, Hermans C, Lambert C. Optimal management of hemophilic arthropathy and hematomas. J Blood Med Dove Press. 2014;5:207-18.

5. World Federation of Hemophilia. Guidelines for the Management of Hemophilia [Internet]. 2012. Available from: https://www1.wfh.org/ publication/files/pdf-1472.pdf.

6. Mulder K, Llinás A. The target joint. Haemophilia. 2004;10(Suppl 4):152-6.

7. Monahan PE, Baker JR, Riske B, Soucie JM. Physical functioning in boys with hemophilia in the U.S. Am J Prev Med. 2011:41:S360-8.

8. Bladen M, Main E, Hubert N, Koutoumanou E, Liesner R, Khair K. Factors affecting the Haemophilia joint health score in children with severe haemophilia. Haemophilia. 2013;19:626-31.

9. Soucie JM, Wang C, Siddiqi A, Kulkarni R, Recht M, Konkle BA, et al. The longitudinal effect of body adiposity on joint mobility in young males with Haemophilia a. Haemophilia. 2011;17:196-203.

10. Manco-Johnson MJ, Soucie JM, Gill JC. Prophylaxis usage, bleeding rates and joint outcomes of hemophilia 1999 - 2010: a surveillance project. Blood. 2017;129:2368-75.

11. Santagostino E, Auerswald G, Benson G, Dolan G, Jiménez-Yuste V, Lambert $T$, et al. Switching treatments in haemophilia: is there a risk of inhibitor development. Eur J Haematol. 2015;94:284-9. Wiley-Blackwell

12. Bohn RLRL, Aledort LM, Putnam K, Ewenstein B, Mogun H, Avorn JJ. The economic impact of factor VIII inhibitors in patients with haemophilia. Haemophilia. 2004;10:63-8.

13. Coppola A, Cerbone AM, Mancuso G, Mansueto MF, Mazzini C, Zanon E. Confronting the psychological burden of haemophilia. Haemophilia. 2011; 17:21-7.

14. Kodra Y, Cavazza M, Schieppati A, De Santis M, Armeni P, Arcieri R, et al. The social burden and quality of life of patients with haemophilia in Italy. Blood Transfus. 2014;12(Suppl 3):s567-75. SIMTI Servizi

15. Klamroth R, Pollmann H, Hermans C, Faradji A, Yarlas AS, Epstein JD, et al. The relative burden of haemophilia a and the impact of target joint development on health-related quality of life: results from the ADVATE post-authorization safety surveillance (PASS) study. Haemophilia. 2011;17: 412-21

16. Camp C, Carroll L, Hughes D, Burke T, OHara J. An Introduction to "The Cost of Haemophilia across Europe - a Socioeconomic Survey " (CHESS). Poster presented at WFH 2016 World Congress. 
17. Knight $\mathrm{C}$. Health economics of treating haemophilia a with inhibitors. Haemophilia. 2005;11(Suppl 1):11-7.

18. Brazier J, Ratcliffe J, Tsuchiya A, Salomon J. Measuring and valuing health benefits for economic evaluation. Second. Oxford: Oxford University Press; 2016.

19. Koopmanschap M. Coping with type II diabetes: the patient's perspective. Diabetologia. 2002;45:S18-22.

20. Konkle BA, Ebbesen LS, Erhardtsen E, Bianco RP, Lissitchkov T, Rusen L, et al. Randomized, prospective clinical trial of recombinant factor Vlla for secondary prophylaxis in hemophilia patients with inhibitors. J Thromb Haemost. 2007;5:1904-13. Blackwell Publishing Ltd

21. Glick HA, Doshi JA, Sonnad SS, Polsky D. Economic evaluation in clinical trials. Oxford: Oxford University Press; 2014.

22. Mihaylova B, Briggs A, O'Hagan A, Thompson SG. Review of statistical methods for analysing healthcare resources and costs. Health Econ. 2011;20: 897-916.

23. Coughlan D, Yeh ST, Neill CO, Frick KD. Evaluating direct medical expenditures estimation methods of adults using the medical expenditure panel Survey : an example focusing on head and neck cancer. Value Heal. 2014;17:90-7. Elsevier

24. Willan A, Briggs A. Statistical analysis of cost-effectiveness data. Chichester: John Wiley \& Sons Ltd; 2006.

25. Manning WG, Mullahy J. Estimating log models: to transform or not to transform? J Health Econ. 2001;20:461-94.

26. McCaffrey N, Kaambwa B, Currow DC, Ratcliffe J. Health-related quality of life measured using the EQ-5D-5L: south Australian population norms. Heal Qual Life Outcomes. 2016:14:133.

27. StataCorp. 2013. Stata Statistical Software: Release 13. College Station, TX: StataCorp LP. https://www.stata.com/support/faqs/resources/citing-softwaredocumentation-faqs/.

28. Royal S, Schramm W, Berntorp E, Giangrande P, Gringeri A, Ludlam C, et al. Quality-of-life differences between prophylactic and on-demand factor replacement therapy in European haemophilia patients. Haemophilia. 2002; 8:44-50.

29. Rossbach HC. Review of antihemophilic factor injection for the routine prophylaxis of bleeding episodes and risk of joint damage in severe hemophilia a. Vasc Health Risk Manag. 2010;6:59-68.

30. Walsh M, Macgregor D, Stuckless S, Barrett B, Kawaja M, Scully M-F. Healthrelated quality of life in a cohort of adult patients with mild hemophilia a. J Thromb Haemost. 2008;6:755-61.

31. Siboni SM, Mannucci PM, Gringeri A, Franchini M, Tagliaferri A, Ferretti M, et al. Health status and quality of life of elderly persons with severe hemophilia born before the advent of modern replacement therapy. $J$ Thromb Haemost. 2009;7:780-6.

32. Blanchette VS, Key NS, Ljung LR, Manco-Johnson MJ, van den Berg HM, Srivastava A, et al. Definitions in hemophilia: communication from the SSC of the ISTH. J Thromb Haemost. 2014;12:1935-9.

33. Goddard NJ, Mann H. Diagnosis of haemophilic synovitis. Haemophilia. 2007;13:14-9.

34. O'Hara J, Walsh S, Camp C, Mazza G, Carroll L, Hoxer C, et al. The relationship between target joints and direct resource use in severe haemophilia. Health. Econ Rev. 2018;8:1. SpringerOpen

35. Fischer $\mathrm{K}$, van der Bom JG, van den Berg HM. Health-related quality of life as outcome parameter in haemophilia treatment. Haemophilia. 2003;9(Suppl 1):75-81. discussion 82

36. Agborsangaya CB, Lahtinen $\mathrm{M}$, Cooke $\mathrm{T}$, Johnson JA. Comparing the EQ-5D $3 \mathrm{~L}$ and $5 \mathrm{~L}$ : measurement properties and association with chronic conditions and multimorbidity in the general population. Health Qual Life Outcomes. 2014;12:74.

\section{Ready to submit your research? Choose BMC and benefit from:}

- fast, convenient online submission

- thorough peer review by experienced researchers in your field

- rapid publication on acceptance

- support for research data, including large and complex data types

- gold Open Access which fosters wider collaboration and increased citations

- maximum visibility for your research: over $100 \mathrm{M}$ website views per year

At BMC, research is always in progress.

Learn more biomedcentral.com/submissions 